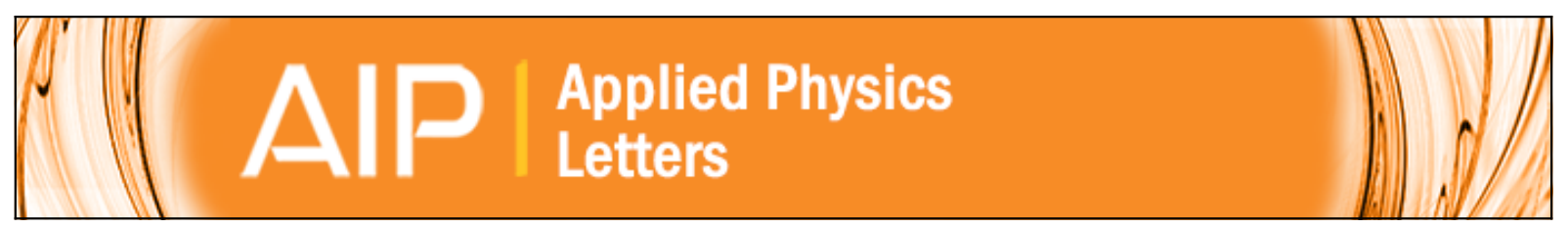

\title{
Single-step colloidal quantum dot films for infrared solar harvesting
}

Amirreza Kiani, Brandon R. Sutherland, Younghoon Kim, Olivier Ouellette, Larissa Levina, Grant Walters, Cao-

Thang Dinh, Mengxia Liu, Oleksandr Voznyy, Xinzheng Lan, Andre J. Labelle, Alexander H. Ip, Andrew Proppe, Ghada H. Ahmed, Omar F. Mohammed, Sjoerd Hoogland, and Edward H. Sargent

Citation: Applied Physics Letters 109, 183105 (2016); doi: 10.1063/1.4966217

View online: http://dx.doi.org/10.1063/1.4966217

View Table of Contents: http://scitation.aip.org/content/aip/journal/apl/109/18?ver=pdfcov

Published by the AIP Publishing

\section{Articles you may be interested in}

Low-cost high-haze films based on $\mathrm{ZnO}$ nanorods for light scattering in thin c-Si solar cells

Appl. Phys. Lett. 106, 013901 (2015); 10.1063/1.4905389

Influence of the absorber layer thickness and rod length on the performance of three-dimensional nanorods thin film hydrogenated amorphous silicon solar cells

J. Appl. Phys. 113, 163106 (2013); 10.1063/1.4803045

CdS quantum dots grown by in situ chemical bath deposition for quantum dot-sensitized solar cells J. Appl. Phys. 110, 044313 (2011); 10.1063/1.3624944

Determination of limiting factors of photovoltaic efficiency in quantum dot sensitized solar cells: Correlation between cell performance and structural properties

J. Appl. Phys. 108, 064310 (2010); 10.1063/1.3477194

Quantum-dot-sensitized solar cells: Assembly of CdS-quantum-dots coupling techniques of self-assembled monolayer and chemical bath deposition

Appl. Phys. Lett. 90, 143517 (2007); 10.1063/1.2721373

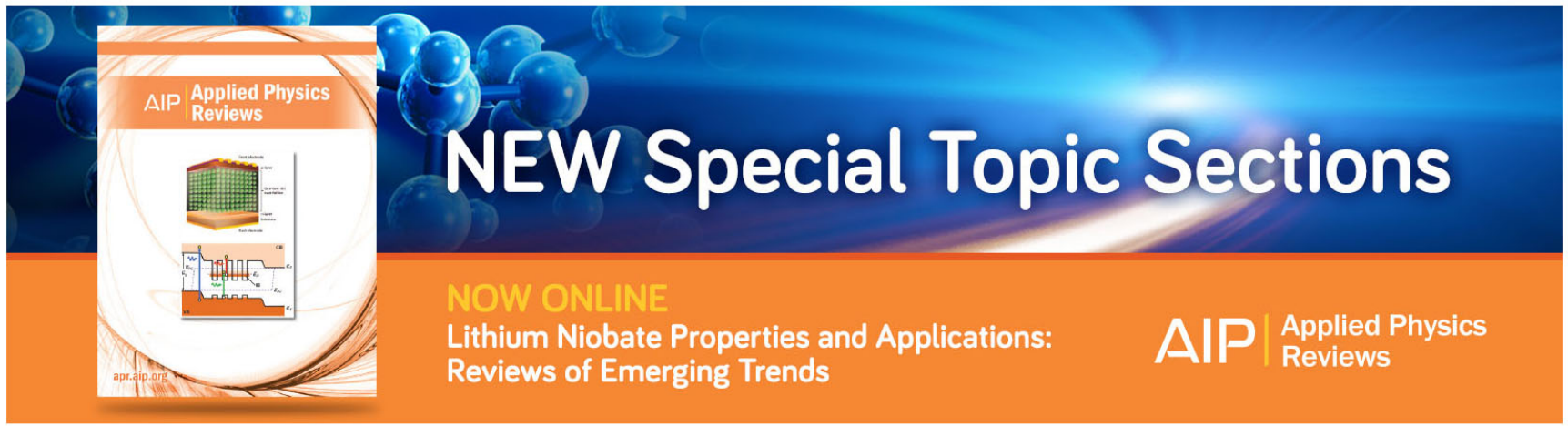




\title{
Single-step colloidal quantum dot films for infrared solar harvesting
}

\author{
Amirreza Kiani, ${ }^{1, a)}$ Brandon R. Sutherland, ${ }^{1, a)}$ Younghoon Kim, ${ }^{1}$ Olivier Ouellette, ${ }^{1}$ \\ Larissa Levina, ${ }_{1}^{1}$ Grant Walters, ${ }^{1}$ Cao-Thang Dinh, ${ }^{1}$ Mengxia Liu, ${ }^{1}$ Oleksandr Voznyy, ${ }^{1}$ \\ Xinzheng Lan, ${ }^{1}$ Andre J. Labelle, ${ }^{1}$ Alexander H. Ip, ${ }^{1}$ Andrew Proppe, ${ }^{1}$ Ghada H. Ahmed, ${ }^{2}$ \\ Omar F. Mohammed, ${ }^{2}$ Sjoerd Hoogland, ${ }^{1}$ and Edward H. Sargent ${ }^{1, b)}$ \\ ${ }^{1}$ Department of Electrical and Computer Engineering, University of Toronto, 10 King's College Road, \\ Toronto, Ontario M5S 3G4, Canada \\ ${ }^{2}$ Division of Physical Sciences and Engineering, Solar and Photovoltaics Engineering Research Center, \\ King Abdullah University of Science and Technology (KAUST), Thuwal 23955-6900, Kingdom of Saudi Arabia
}

(Received 8 July 2016; accepted 7 October 2016; published online 31 October 2016)

\begin{abstract}
Semiconductors with bandgaps in the near- to mid-infrared can harvest solar light that is otherwise wasted by conventional single-junction solar cell architectures. In particular, colloidal quantum dots (CQDs) are promising materials since they are cost-effective, processed from solution, and have a bandgap that can be tuned into the infrared (IR) via the quantum size effect. These characteristics enable them to harvest the infrared portion of the solar spectrum to which silicon is transparent. To date, IR CQD solar cells have been made using a wasteful and complex sequential layer-by-layer process. Here, we demonstrate $\sim 1 \mathrm{eV}$ bandgap solar-harvesting CQD films deposited in a single step. By engineering a fast-drying solvent mixture for metal iodide-capped CQDs, we deposited active layers greater than $200 \mathrm{~nm}$ in thickness having a mean roughness less than $1 \mathrm{~nm}$. We integrated these films into infrared solar cells that are stable in air and exhibit power conversion efficiencies of 3.5\% under illumination by the full solar spectrum, and $0.4 \%$ through a simulated silicon solar cell filter. Published by AIP Publishing. [http://dx.doi.org/10.1063/1.4966217]
\end{abstract}

Silicon photovoltaic cells comprise over $90 \%$ of installed commercial solar modules worldwide. ${ }^{1}$ The increasing adoption of silicon solar cells has been driven by an impressive rate of decrease in the cost of solar energy production from 76 $\$ / \mathrm{W}_{\mathrm{p}}$ in 1970 to $0.15 \$ / \mathrm{W}_{\mathrm{p}}$ in 2015 . $^{2}$ As this cost continues to diminish, solar energy production will continue to become an increasingly viable solution to meet energy demands. It is estimated that solar electricity could meet $20 \%$ of the world's energy consumption by $2030 .^{2}$ It is important to note that this increase in solar energy consumption is largely driven by cost reductions, which are now leveling off. There is therefore a substantial need for new solar solutions at low costs that augment the efficiency of silicon-based solar cells.

Over a period of two decades, the record research efficiency of silicon-based solar cells has seen an increase of $2 \%$ (absolute percent or power points). ${ }^{3}$ This could be further enhanced by generating power from solar light that is not absorbed by silicon cells. Up to 7 power points are available to be added to a bifacial heterojunction silicon solar cell using power in the infrared (IR) portion of the solar spectrum. ${ }^{4}$

Colloidal quantum dots (CQDs) are emerging thirdgeneration solar materials. They are processed from the solution phase, and their bandgap can be tuned via the quantum size effect. They are capable of absorbing IR light (beyond $1 \mu \mathrm{m})$ and are also compatible with large-area, massmanufacturing deposition techniques such as spray-coating. ${ }^{5}$ Solar cells based on $1.3 \mathrm{eV}$ bandgap CQDs have achieved a record full-spectrum certified efficiency of $11.3 \% .^{3}$ Recently, $1 \mathrm{eV}$ bandgap CQD cells with the efficiencies of $7.3 \%$ full

\footnotetext{
${ }^{\text {a) }}$ A. Kiani and B. R. Sutherland contributed equally to this work.

b) Author to whom correspondence should be addressed. Electronic mail: ted.sargent@utoronto.ca.
}

spectrum and $0.8 \%$ through a simulated silicon filter have been demonstrated. ${ }^{4}$ In that work, the active layer was deposited sequentially in a layer-by-layer process, consisting of 12 layers, each with one ligand-exchange treatment and two washing steps. This multi-step processing makes manufacturing complex, and, additionally, the material is wasted with each layer deposited and treated. Furthermore the CQD active layer used 3-mercaptapropionic acid as a ligand, and this is known to be unstable under air-storage without encapsulation. ${ }^{6}$ In this work, we sought to address these issues by developing $1 \mathrm{eV}$ bandgap CQD solar cells that benefit from airstable iodide passivation and where the active layer is deposited in a single step with no post-treatment. To date, there are no reports of solution-exchanged $1 \mathrm{eV}$ bandgap CQD solar harvesting films.

To deposit IR CQDs in a single step, we first required a nanocrystal colloid stabilized using short conductive ligands on the nanoparticle surface. To achieve this, we improved upon an anti-solvent phase-boundary exchange method used for $1.3 \mathrm{eV}$ CQDs. ${ }^{7,8}$ In this process, oleic-acid (OA)-capped PbS CQDs (OA-PbS) dispersed in octane are mixed with halide precursors that are dissolved in $\mathrm{N}, \mathrm{N}-$ dimethylformamide (DMF). Previous work on anti-solvent phase boundary exchanges had used methylammonium iodide $^{7}$ and methylammonium lead tri-iodide for the ligandexchange process. ${ }^{8}$ Here, we use lead iodide $\left(\mathrm{PbI}_{2}\right)$ that can also act as a ligand, which has recently been shown to increase short-circuit current in a solid-state treatment. ${ }^{9}$ Further, the iodide ligands have been demonstrated to be stable under air storage. ${ }^{10,11}$

The OA-PbS CQDs were synthesized following a previously published method. ${ }^{12}$ They have a first absorption peak at approximately $1300 \mathrm{~nm} .0 .5 \mathrm{~mL}$ of OA-PbS $(50 \mathrm{mg} / \mathrm{ml})$ in 
octane was added to a mixture of $4.5 \mathrm{~mL}$ of octane, $100 \mathrm{mg}$ of $\mathrm{PbI}_{2}$, and $30 \mathrm{mg}$ of ammonium acetate $\left(\mathrm{C}_{2} \mathrm{H}_{3} \mathrm{O}_{2} \mathrm{NH}_{4}\right)$ dissolved in $3 \mathrm{~mL}$ of DMF. After vortexing this mixture, we observed a favourable exchange from $\mathrm{OA}-\mathrm{PbS}$ to $\mathrm{PbI}_{2}-$ capped $\mathrm{PbS}$ CQDs $\left(\mathrm{PbI}_{2}-\mathrm{PbS}\right)$, with the CQDs entering the DMF phase (Figure 1(a)).

To form thick single-step films from these solar inks, the CQDs must be redispersed in a fast-drying solvent at high concentrations (greater than $200 \mathrm{mg} / \mathrm{ml}$ ) after being precipitated from DMF. Typically, n-butylamine (BTA) is used as a final solvent for $\sim 1.3 \mathrm{eV}$ halide-capped solution-exchanged CQDs. ${ }^{7,8}$ We first attempted to form a stable colloid of $\sim 1 \mathrm{eV}$ $\mathrm{PbI}_{2}-\mathrm{PbS}$ nanocrystals in BTA. However, we found that a large proportion of the CQDs instantly agglomerated and could not be redispersed in the solvent. Consequently, we were unable to concentrate the $\mathrm{PbI}_{2}-\mathrm{PbS}$ CQDs sufficiently in BTA to form films at solar-relevant thicknesses. We observed similar agglomeration of $\sim 1 \mathrm{eV}$ CQDs capped with $\mathrm{MAPbI}_{3}$.

The shape of PbS CQDs is strongly size-dependent. As the diameter of the CQD is increased to approximately $4 \mathrm{~nm}$, the shape changes from octahedron to cuboctahedron. ${ }^{13}$ This $\mathrm{PbS}$ diameter corresponds to a bandgap of approximately $1.1 \mathrm{eV}{ }^{14,15}$ Therefore, when size-tuning PbS CQDs to $1 \mathrm{eV}$ for infrared solar harvesting, there is a dramatic change in the surface of the nanocrystal. It has been previously demonstrated that it becomes increasingly difficult to stabilize $\mathrm{PbS}$ CQDs as they increase in size. OA-capped CQDs exhibit absorption-broadening as the diameter is increased from 3.3 to $8.5 \mathrm{~nm} .{ }^{16}$ Solution-exchanged $8.7 \mathrm{~nm}$ CQDs capped with $\mathrm{MAPbI}_{3}$ have shown size distributions with agglomeration tails, whereas it is not present in similarly treated $3.8 \mathrm{~nm}$ CQDs, and both $8.7 \mathrm{~nm}$ and $4.5 \mathrm{~nm} \mathrm{MAPbI}_{3}$-capped CQDs have shown broader red-tails in solution absorption compared with a $3.8 \mathrm{~nm}$ control. ${ }^{17}$ We have observed that these issues of agglomeration become a limiting factor especially when trying to stabilize solution-exchanged $\mathrm{PbS}$ CQDs at high concentrations for single-step fabrication. To overcome this, we needed to develop processes which were tailored to the colloidal stabilization of large solution-exchanged $\sim 1 \mathrm{eV}$ CQDs in concentrated fast-drying solvents.
We hypothesized that we could improve the colloidal stability of these CQDs by using a longer-chain amine solvent. Longer-chain amines as ligands have been demonstrated to aid colloidal stabilization and improve surface passivation in CdSe CQDs. ${ }^{18,19}$ We found that hexylamine (HXA) could completely redisperse the quantum dots. However, due to its higher boiling point, the resulting films were comparable in thickness to those made from BTA. To overcome this, we mixed HXA with methyl-ethyl-ketone (MEK), a lower-boiling point solvent. While pure-MEK does not dissolve the CQDs at all, a mix of 20\% HXA and $80 \%$ MEK is sufficient to redisperse the CQDs fully. This ratio is used for all subsequent characterization unless otherwise specified. We fabricated films of $\mathrm{PbI}_{2}-\mathrm{CQDs}$ in $\mathrm{BTA}$, HXA, and HXA+MEK (20:80\%) via spin-coating at $2500 \mathrm{rpm}$. The concentration of the $\mathrm{PbI}_{2}-\mathrm{CQDs}$ in HXA and HXA+MEK was $200 \mathrm{mg} / \mathrm{ml}$, while $\mathrm{PbI}_{2}-\mathrm{CQDs}$ in BTA formed a supersaturated solution at an estimated concentration of less than $100 \mathrm{mg} / \mathrm{ml}$. Atomic force microscopy (Asylum Research Cypher S) operated in tapping mode with Olympus AC240TM-R3 probes was used to measure the surface morphology and the film thickness (Figures 1(b) and 1(c)). The mixed HXA+MEK solvent dramatically reduced the root-mean-squared (RMS) surface roughness to below $1 \mathrm{~nm}$ compared to $60 \mathrm{~nm}$ (HXA as a solvent) and $34 \mathrm{~nm}$ (BTA as a solvent).

We then set out to characterize the spectroscopic properties of the CQDs as dispersed in HXA+MEK. We observed only a minimal change in the absorption (PerkinElmer Lambda 950) and photoluminescence (PL, Horiba FluoroLog-3) spectra of CQDs dispersed in octane (OA ligands), DMF ( $\mathrm{PbI}_{2}$ ligands), and HXA+MEK ( $\mathrm{PbI}_{2}$ ligands) (Figure 2(a)). The Stokes shift of these materials in the solution-phase is approximately $40 \mathrm{~nm}$ in octane and DMF and less than $35 \mathrm{~nm}$ in HXA+MEK. These shifts are comparable to previouslyreported values for $\mathrm{PbS}$ CQDs with this bandgap. ${ }^{20}$ The absorption and PL in the film (Figure 2(b)) are red-shifted by approximately $60 \mathrm{~nm}$, which suggests electronic coupling among the CQDs in the solid state. X-ray photoelectron spectroscopy (XPS, PHI-5500) reveals a strong signature for (a)

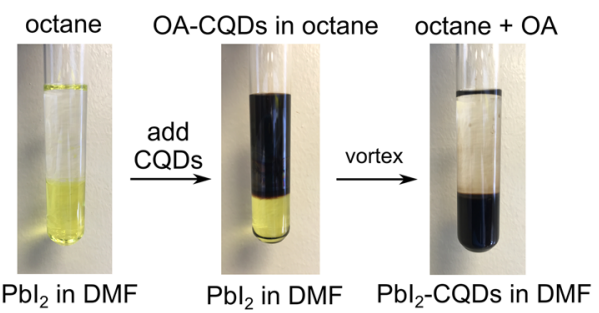

(c)

$$
\mathrm{Pbl}_{2} \text { in } \mathrm{DMF}
$$

$\mathrm{Pbl}_{2}$ in $\mathrm{DMF}$

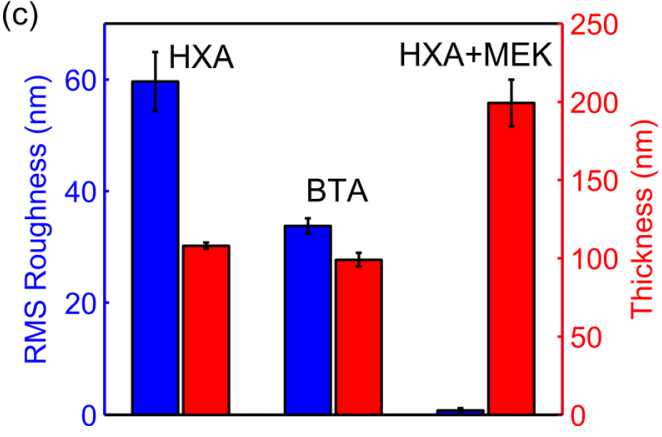

(b)

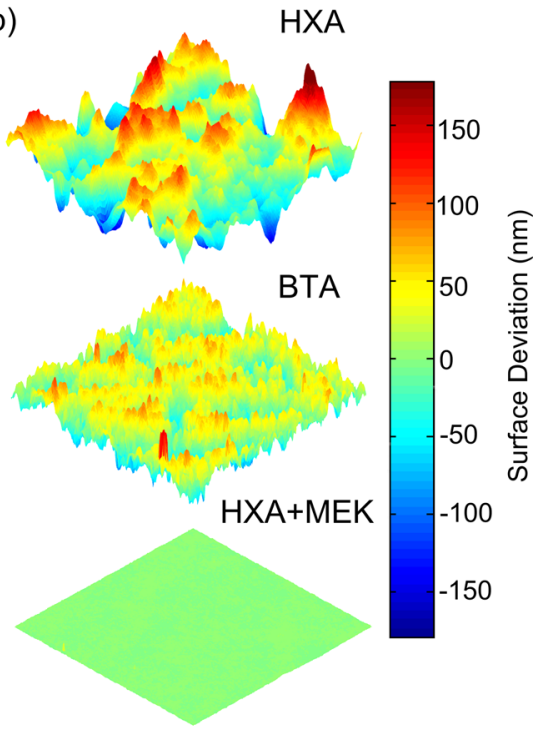

FIG. 1. Solvent optimization for the CQD inks. (a) Infrared $\mathrm{PbS}$ CQD solution-exchange process from OAligands to $\mathrm{PbI}_{2}$ ligands. (b) The AFM surface topology of a $10 \mu \mathrm{m}^{2}$ square area for films processed from HXA, BTA, and HXA+MEK. (c) RMS roughness and thickness of fabricated films. We observe that HXA+MEK yields the greatest film thickness and the smallest RMS roughness. 

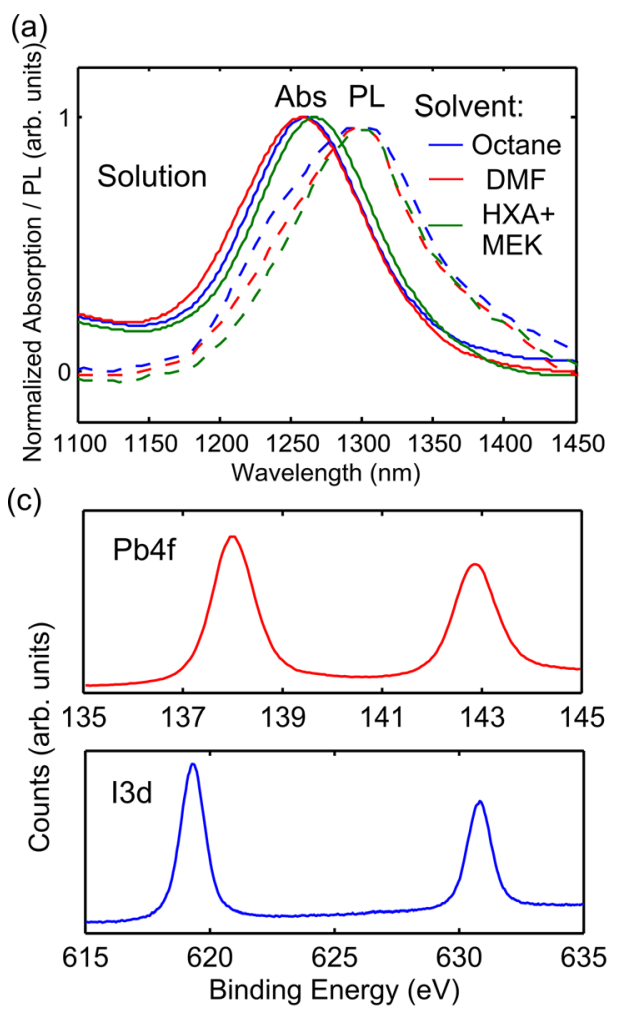

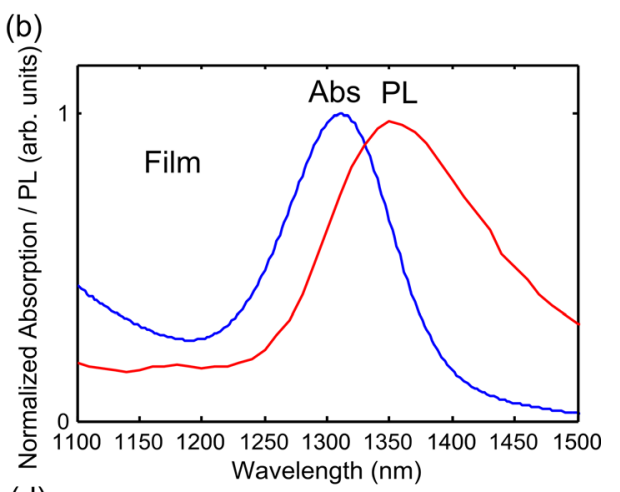

(d)

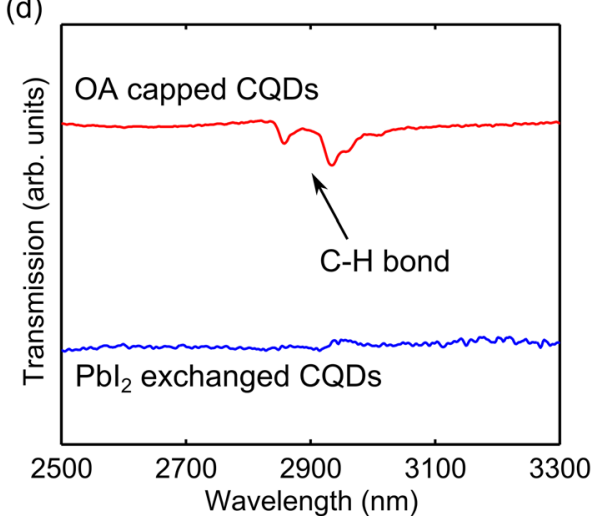

FIG. 2. The spectroscopic properties of solution-exchanged IR $\mathrm{PbS}$ CQDs capped with $\mathrm{PbI}_{2}$ ligands. (a) Normalized absorption (solid line) and PL (dashed line) in the solution phase for oleic acid capped CQDs in octane and for $\mathrm{PbI}_{2}$ capped CQDs in DMF and HXA+MEK. (b) Normalized film absorption and PL for the CQDs in HEX+MEK. The PL spectra in solution and film have a Savitzky-Golay filter of degree 2 and span $10 \mathrm{~nm}$ applied. (c) XPS surveys of Pb4f (top) and I3d (bottom) of $\mathrm{PbI}_{2}$ exchanged CQD films deposited from the HEX+MEK final solvent. (d) The FTIR scan of the C-H bond. We observe a reduction of the $\mathrm{C}-\mathrm{H}$ signal in the $\mathrm{PbI}_{2}$ exchanged CQDs compared to OA-CQDs. iodide, suggesting good incorporation of iodide into the final film (Figure 2(c)). A Pb:I ratio is estimated at 0.7, comparable to previous solution-exchanges with methylammonium iodide. ${ }^{7}$ The Fourier-transform infrared (FTIR) spectroscopy analysis (Bruker Tensor 27) reveals a quenching of the $\mathrm{C}-\mathrm{H}$ bond for the $\mathrm{PbI}_{2}$ exchanged CQDs relative to oleic-acid capped CQDs (Figure 2(d)). The XPS and FTIR results jointly suggest that oleic acid has been replaced by iodine during the exchange process.

We fabricated photovoltaic devices using these singlestep CQD films as the active layer (Figure 3(a)). Lowconductivity ITO (Delta Technologies, 80-90 $\Omega$ /square) was used to ensure minimal absorption in the infrared. We deposited $100 \mathrm{~nm} \mathrm{ZnO}-\mathrm{NPs}$ using a previously-reported procedure. $^{21} \mathrm{PbI}_{2}-\mathrm{PbS}$ CQDs were spin-coated $(250 \mathrm{mg} / \mathrm{ml}$ at $2500 \mathrm{rpm}$ ) onto ZnO-NPs. The two layers of a PbS-EDT hole transporting layer were deposited using a previously reported method, ${ }^{21}$ followed by $120 \mathrm{~nm}$ of thermally evaporated $\mathrm{Au}$ (Angstrom Engineering Åmod).

Cross-sectional scanning electron microscopy (SEM, FEI Environmental SEM, $10 \mathrm{kV}$ ) reveals a distinct region of CQDs of $\sim 350 \mathrm{~nm}$ in thickness (Figure 3(b)). The fullspectrum and $1100 \mathrm{~nm}$ long-pass (Thorlabs FEL1100 filter) current-voltage characteristics under AM1.5G illumination (ScienceTech, measured to be within Class A specifications) each exhibit minimal hysteresis between forward and reverse bias sweeps (Figure 3(c)). A correction factor of 1.5 accounting for the spectral mismatch between the solar simulator and true AM1.5G beyond $1100 \mathrm{~nm}$ was obtained using a previously reported method. ${ }^{4}$ Spectral external quantum efficiency (EQE) was measured using a calibrated $450 \mathrm{~W}$ xenon lamp through a monochromator. The incident beam was modulated at $220 \mathrm{~Hz}$ and collimated. From the spectral EQE, we confirmed that the integrated $\mathrm{J}_{\mathrm{sc}}$ matches the AM1.5 $\mathrm{J}_{\mathrm{sc}}$ (both full spectrum and filtered) and demonstrates 40\% EQE at the excitonic peak (Figure 3(d)). The best-performing devices exhibit full-spectrum and filtered power conversion efficiency (PCE) of $3.5 \%$ and $0.4 \%$, respectively (Figure 3(e)). The devices exhibit no decrease in PCE when stored in air and periodically tested over a 3-month period, Figure S1. The average PCE of an identically-prepared set of these devices illuminated through an $1100 \mathrm{~nm}$ filter (based on 20 devices over 7 substrates) is $0.32 \% \pm 0.04 \%$.

In summary, we have demonstrated a tailored solution exchange process for infrared-absorbing CQDs $(\sim 1 \mathrm{eV}$ bandgap). It enables the realization of manufacturable devices that can augment the performance of high-efficiency PV materials whose spectral cutoff lies at $1100 \mathrm{~nm}$ or shorter. We developed a solution-exchange protocol that enabled us to deposit, in a single step, an active layer with greater than $200 \mathrm{~nm}$ thickness and a RMS roughness below $1 \mathrm{~nm}$. The strategy relied on the combination of hexylamine and MEK as co-solvents. The process resulted in an air-stable IR PV device capable of adding 0.4 power points to a bifacial silicon cell. This work showcases the capability of solution-processed quantum dot devices to enhance silicon solar cells. It provides a customized chemical approach for $1 \mathrm{eV}$ bandgap CQDs with improved manufacturability compared to the previous layerby-layer approaches.

There remain challenges that must be addressed to improve the performance of IR CQDs in sensitizing silicon photovoltaics. Methods of forming IR CQD solids with increased diffusion lengths must be developed to enable thicker active layers resulting in complete absorption of incident IR light. Strategies that photonically trap IR light in the active layer can leverage the high dielectric constant of the IR-bandgap CQD solids. ${ }^{22}$ It is also important to address the band alignment between the CQD active layer and the 
(a)

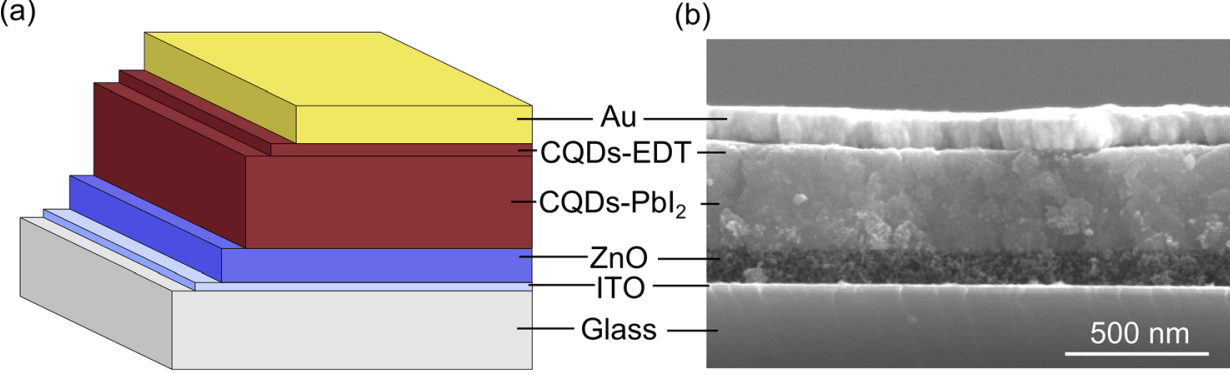

(c)

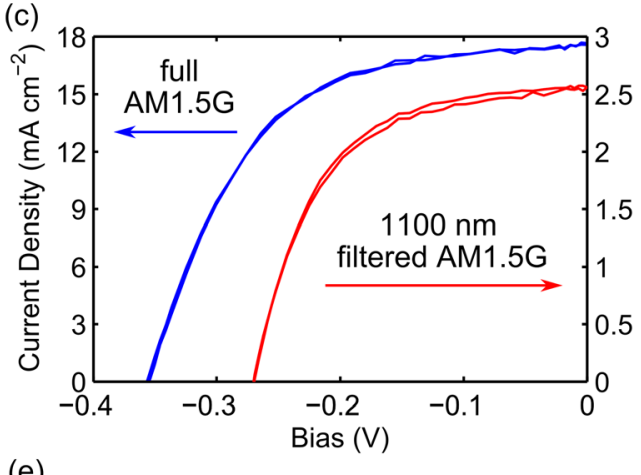

(d)

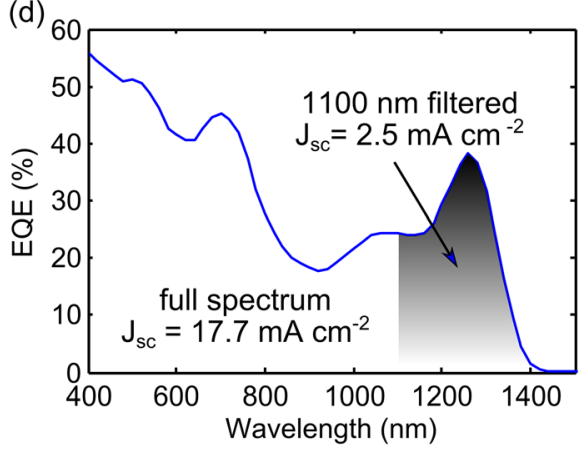

FIG. 3. Solar cells based on solution exchanged IR $\mathrm{PbI}_{2}$-capped PbS CQDs dispersed in HXA+MEK as the final solvent. (a) Device schematic. (b) Crosssectional SEM of a representative device. (c) The AM1.5G performance of a champion cell, both full-spectrum (left curve) and through an $1100 \mathrm{~nm}$ longpass filter (right curve). (d) Spectral $\mathrm{EQE}$ of the same cell. The integrated $\mathrm{J}_{\mathrm{sc}}$ matches the measured AM1.5G J $\mathrm{J}_{\mathrm{sc}}$ both full-spectrum and through the $1100 \mathrm{~nm}$ filter. The EQE spectrum has a Savitzky-Golay filter of degree 2 and span $30 \mathrm{~nm}$ applied. (e) Performance table summary of a champion cell. The device exhibits a near equivalent performance after 3 months in air storage.

(e)

\begin{tabular}{|c|c|c|c|c|c|}
\hline & & $\mathrm{J}_{\mathrm{sc}}\left(\mathrm{mA} \mathrm{cm} \mathrm{cm}^{-2}\right)$ & $V_{o c}(V)$ & FF (\%) & PCE (\%) \\
\hline & Full-spectrum & 17.4 & 0.36 & 55 & 3.5 \\
\hline & $1100 \mathrm{~nm}$ filtered & 2.6 & 0.27 & 57 & 0.4 \\
\hline \multirow{2}{*}{$\begin{array}{l}\text { After } 3 \text { months } \\
\text { air storage }\end{array}$} & Full-spectrum & 18.1 & 0.38 & 54 & 3.7 \\
\hline & $1100 \mathrm{~nm}$ filtered & 2.4 & 0.29 & 58 & 0.4 \\
\hline
\end{tabular}

electron-accepting electrode: halide treatment of CQDs, while beneficial for optoelectronic properties, results in a deeper conduction band; ${ }^{23}$ this makes injection into shallow work-function electron accepting electrodes, such as unmodified $\mathrm{TiO}_{2}$ or $\mathrm{ZnO}$, inefficient, as evidenced by PL quenching measurements (Figure S2). As a result, new deep work function electrode materials with sufficient electron mobility and the capability of being doped to heavily n-type will be required for further progress.

See supplementary material for the effect of $\mathrm{ZnO}$ on the carrier extraction for different bandgap CQDs and solar cell stability data.

This publication is based in part on the work supported by the Ontario Research Fund-Research Excellence Program, ORF \#07-042.

1“Global Market Outlook for Solar Power 2015-2019" (SolarPower, Europe, 2015).

${ }^{2}$ J. D. Farmer and F. Lafond, Res. Policy 45, 647 (2016).

${ }^{3}$ NREL Efficiency Chart, 2016.

${ }^{4}$ A. H. Ip, A. Kiani, I. J. Kramer, O. Voznyy, H. F. Movahed, L. Levina, M. M. Adachi, S. Hoogland, and E. H. Sargent, ACS Nano 9, 8833 (2015).

${ }^{5}$ I. J. Kramer, J. C. Minor, G. Moreno-Bautista, L. Rollny, P. Kanjanaboos, D. Kopilovic, S. M. Thon, G. H. Carey, K. W. Chou, D. Zhitomirsky, A. Amassian, and E. H. Sargent, Adv. Mater. 27, 116 (2015).

${ }^{6}$ A. H. Ip, A. J. Labelle, and E. H. Sargent, Appl. Phys. Lett. 103, 263905 (2013).
${ }^{7}$ Z. Ning, H. Dong, Q. Zhang, O. Voznyy, and E. H. Sargent, ACS Nano 8, 10321 (2014).

${ }^{8}$ Z. Yang, A. Janmohamed, X. Lan, F. P. García de Arquer, O. Voznyy, E. Yassitepe, G.-H. Kim, Z. Ning, X. Gong, R. Comin, and E. H. Sargent, Nano Lett. 15, 7539 (2015).

${ }^{9}$ R. W. Crisp, D. M. Kroupa, A. R. Marshall, E. M. Miller, J. Zhang, M. C. Beard, and J. M. Luther, Sci. Rep. 5, 9945 (2015).

${ }^{10}$ C.-H. M. Chuang, P. R. Brown, V. Bulović, and M. G. Bawendi, Nat. Mater. 13, 796 (2014).

${ }^{11}$ G.-H. Kim, F. P. García de Arquer, Y. J. Yoon, X. Lan, M. Liu, O. Voznyy, Z. Yang, F. Fan, A. H. Ip, P. Kanjanaboos, S. Hoogland, J. Y. Kim, and E. H. Sargent, Nano Lett. 15, 7691 (2015).

${ }^{12}$ M. A. Hines and G. D. Scholes, Adv. Mater. 15, 1844 (2003).

${ }^{13}$ H. Choi, J.-H. Ko, Y.-H. Kim, and S. Jeong, J. Am. Chem. Soc. 135, 5278 (2013).

${ }^{14}$ I. Kang and F. W. Wise, J. Opt. Soc. Am. B 14, 1632 (1997).

${ }^{15}$ D. Segets, J. M. Lucas, R. N. Klupp Taylor, M. Scheele, H. Zheng, A. P. Alivisatos, and W. Peukert, ACS Nano 6, 9021 (2012).

${ }^{16} \mathrm{~J}$. Jasieniak, M. Califano, and S. E. Watkins, ACS Nano 5, 5888 (2011).

${ }^{17}$ D. N. Dirin, S. Dreyfuss, M. I. Bodnarchuk, G. Nedelcu, P. Papagiorgis, G. Itskos, and M. V. Kovalenko, J. Am. Chem. Soc. 136, 6550 (2014).

${ }^{18}$ N. C. Anderson, M. P. Hendricks, J. J. Choi, and J. S. Owen, J. Am. Chem. Soc. 135, 18536 (2013).

${ }^{19}$ Z. M. Norman, N. C. Anderson, and J. S. Owen, ACS Nano 8, 7513 (2014).

${ }^{20}$ M. C. Weidman, M. E. Beck, R. S. Hoffman, F. Prins, and W. A. Tisdale, ACS Nano 8, 6363 (2014).

${ }^{21}$ X. Lan, O. Voznyy, A. Kiani, F. P. García de Arquer, A. S. Abbas, G.-H. Kim, M. Liu, Z. Yang, G. Walters, J. Xu, M. Yuan, Z. Ning, F. Fan, P. Kanjanaboos, I. Kramer, D. Zhitomirsky, P. Lee, A. Perelgut, S. Hoogland, and E. H. Sargent, Adv. Mater. 28, 299 (2016).

${ }^{22}$ B. T. Diroll, E. A. Gaulding, C. R. Kagan, and C. B. Murray, Chem. Mater. 27, 6463 (2015).

${ }^{23}$ P. R. Brown, D. Kim, R. R. Lunt, N. Zhao, M. G. Bawendi, J. C. Grossman, and V. Bulović, ACS Nano 8, 5863 (2014). 